\title{
De la Ecovila y Ciudad Eléctrica hasta la aglomeración complexa
}

\section{From the Ecovillages and the Electrical City to the Complex Agglomeration}

Prof. Dr. Javier García-Germán Trujeda

Escuela Técnica Superior de Arquitectura,

Universidad Politécnica de Madrid

Contato: javier@totemarquitectos.com

\begin{abstract}
The discussion on energy and urbanism revolves around two antithetical positions: first the consideration that urban agglomerations must be self-sufficient entities -for instance, the city of Masdar designed by Foster and Partners in Abu Dhabi- and second, an opposite idea that considers that cities should not be isolated but connected to centralized renewable energy networks -for example, the off-shore wind farms currently being championed.

Further analysis unveils that this discussion is unproductive if it focuses either on selfsufficiency or on centralization of renewable electrical supplies. The ideal situation is a balanced mix which takes advantage of both positions. In fact, as there are no definite solutions, it is necessary to have all options open.
\end{abstract}

However, something can be asserted: it is urgent to centre the discussion on energy and urbanism on open thermodynamic systems as, at the end of the day, these are the basis of the functioning of cities. To get to know the energetic reality of a city it is necessary to understand how does an open thermodynamic system work, which is the connection between its structure -form- and its performance, and how do information exchanges affect the matter and energy consumption. This is the only way a discussion on energy and urbanism can be engaged critically.

Keywords: Energy, Self-sufficiency, Open thermodynamic systems, Complexity.

\section{Resumen}

La relación entre ciudad y energía tiende hacia dos situaciones antitéticas: primero, aquella que entiende que las aglomeraciones urbanas deben de ser entes energéticamente autónomos -como es por ejemplo la ciudad de Masdar diseñada por Foster and Partners para Abu Dhabi- y segundo, aquella que entiende que las ciudades no deben estar aislada, sino que deben de estar conectada a redes de energía de rango superior vinculado a la capacidad de soporte de la región sobre la que se enclava - como es el caso de la tendencia hacia los campos eólicos off-shore.

Pero si se estudia este asunto en profundidad, queda claro que esta discusión no se puede limitar al grado de autosuficiencia o al grado de centralización de las redes de suministro. Lo ideal es un mix equilibrado que aproveche las ventajas que ofrece cada situación. La realidad es que existen pocas cuestiones inapelables, resultando necesario tener todas las opciones abiertas.

Sin embargo sí existe una certeza: es necesario que el debate en torno a ciudad y energía se haga partiendo de los sistemas termodinámicos abiertos dado que, al fin y al cabo, éstos están en la base del funcionamiento de las ciudades. Para conocer la realidad energética de una ciudad resulta necesario entender qué es y cómo funciona un sistema termodinámico abierto, cuál es la relación entre su estructura formal y su funcionamiento, y como afectan éstos intercambios de información a su consumo de materia y energía. Únicamente de esta manera se puede establecer un debate serio acerca de la relación entre la ciudad y la energía.

Palavra clave: Energía, Autosuficiencia, Territorio, Sistema termodinámico abierto, Complejidad. 


\section{De la Ecovila y la Ciudade Eléctrica hasta la aglomeracion complexa}

Hoy en día, cuando más de la mitad de la población mundial es urbana, el consumo de energía en las ciudades se eleva al $60 \%$, dato que las delinean como auténticos sumideros energéticos. Y la tendencia es al alza, puesto que se espera que para el año 2030 este consumo haya aumentado en un $15 \%$ ii. Es indudable que será en las grandes ciudades en las que se está concentrando la población mundial, dónde se deban centrar los esfuerzos de reducción del consumo de energía y de emisiones de gases de efecto invernadero. ¿En qué direcciones podemos avanzar?

Durante los últimos años el debate en torno a la ciudad y a la energía ha perfilado dos caminos que han cobrado más fuerza que los demás. Está por un lado la línea que entiende que las aglomeraciones urbanas deben de ser entes autónomos - como es por ejemplo la ciudad de Masdar diseñada por Foster and Partners para Abu Dhabi- que gestionen sus recursos energéticos de modo independiente. $\mathrm{Y}$ por otro lado está la línea que cree que la ciudad no debe estar aislada, sino que debe de estar conectada a redes de energía de escala superior -que responde, por ejemplo, a los proyectos de energías renovables offshore que proliferan en el norte de Europa. Constituyen dos maneras de entender la energía en la ciudad que llevan aparejadas dos modos de concebir la ciudad que abarcan cualquier asunto desde su estructura a su intendencia, conexiones o intercambios.

La primera alternativa, la de las ciudades autosuficientes off the grid, tiene su origen en los años post-1973 cuando la unión de la crisis energética y la crisis ecológica cuestionaron por un lado la enorme dependencia de la tecnología y los sistemas centralizados de suministro energético, y plantearon por otro la posibilidad de regresar a unas formas de vida más cercanas a la naturaleza. Como reacción a esta situación se llevaron a cabo numerosos experimentos de asentamientos autosuficientes, tanto desde su suministro energético como desde su producción de alimentos y otros bienes materiales. Se trataba en la mayoría de los casos del deseo por parte de comunidades hippies de encontrar una vida alternativa a la industrial desarrollando sistemas de autosuficiencia energética -por lo general basados en sistemas low-techcon los que desvincularse de las grandes redes nacionales y del know-how tecnológico. Entre otros casos debemos destacar el solar movement con sus comunas solares en el desierto de Arizona (EE.UU.) o el experimento ARK desarrollado por el New Alchemy Institute en Prince Edward Island (Canadá) iii.

$\mathrm{Si}$ bien estos primeros experimentos han influido de modo decisivo en las recientes ecoaldeas ${ }^{i v}$, han parecido poco extrapolables a las aglomeraciones urbanas de mayor escala e incompatibles con el desarrollo tecnológico. Sin embargo han influido de manera decisiva en gran número de propuestas que han abanderado la unión de autosuficiencia -minimizando intercambios y desplazamientos- y de desarrollo tecnológico. Esto se puede constatar en la manera de entender la relación entre energía y ciudad de las propuestas de Jeremy Rifkinv, gurú de las energías renovables que sigue pensando que la generación de energía solar fotovoltaica unida a tecnologías en desarrollo como la pila de hidrógeno, auguran un futuro de generación doméstica, autosuficiencia y democratización de las fuentes de energía que transformará el urbanismo y la arquitectura. Este entendimiento de la relación ciudad-energía en clave de autosuficiencia también ha alimentando propuestas como la que está desarrollando MVRDV en Logroño (La Rioja, España) o Foster + Partners en Incheon (Corea del Sur): ciudades aisladas y desconectadas -amuralladas en sentido literal y metafórico- que producen todo lo necesario de puertas adentro reduciendo a mínimos los intercambios con el exterior. 
Frente a la propuesta de una ciudad autosuficiente desconectada de su entorno existe una visión opuesta que entiende que una ciudad forma un sistema conectado a las redes que garantizan su supervivencia. De este modo, frente a la idea de una ciudad autosuficiente que minimiza la entropía que genera por concentración de su huella ecológica, ésta otra visión apuesta por una ciudad extendida en el territorio $\mathrm{y}$ que formar parte integral de los sistemas que la alimentan. Esta visión sobre la ciudad tiene su origen en Patrick Geddes quién entendió que la ciudad sólo podía existir en contacto con el hinterland del que dependía. Es clave el concepto de valley section con el que se superaba la idea de ciudad circunscrita a sus límites urbanos para extenderse a toda la región de la que se abastecía, formando un organismo único en constante intercambio de materia, energía e información. Esta aproximación regional fue heredada por una genealogía de pensadores americanos que arrancó con Lewis Mumford, continuó con Ian McHarg, y que con el tiempo ha desembocado en las sofisticadas propuestas de entrelazamiento de los sistemas naturales y urbanos de Richard TT. Forman -sus conocidos land mosaics que dan recomendaciones acerca de los usos, tamaños e interconexiones entre teselas- así como en el trabajo desarrollado por James Corner, Charles Waldheim y otros a través del landscape urbanism.

Este entendimiento de una ciudad ligada a sus sistemas naturales también está en la base de esta segunda manera de entender el suministro de energía como algo vinculado a la capacidad de soporte de la región sobre la que se enclava. El visionario discurso de Geddes no sólo se limitaba al abastecimiento de alimentos o materiales pues también hacía referencia en su libro Cities in Evolution vi a asuntos como el suministro energético de Noruega que ya en 1915 disponía de una red de saltos de agua capaces de interconectar los recursos energéticos regionales a las necesidades de sus ciudades. $\mathrm{Y}$ este mismo concepto forma las bases del plan de energías renovables que se lleva implementando varios años en la Comunidad Autónoma de Navarra (España)vii. En Navarra existen importantes campos eólicos que, como tantos otros, tienen el inconveniente de no generar energía los días poco ventosos y de no poder acumular la energía generada en los días más ventosos. Dado que la orografía de Navarra es acusada, el Plan de Energías Renovables y Eficiencia Energéticaviii renovables contempla la posibilidad acumular los excesos de generación de energía eólica en forma de energía potencial, bombeando agua aguas arriba a sus embalses y pantanos. De este modo se compensan las carencias de la generación eólica en los días poco ventosos con una red de centrales y mini-centrales hidroeléctricas extendidas por toda la región. De este modo, el suministro de energía queda integrado en un sistema energético de orden regional descartando otras soluciones que apuesten por la autosuficiencia de nuevos desarrollos urbanos.

Estas dos perspectivas han dirigido el debate en torno a la ciudad y a la energía durante los últimos años en direcciones opuestas que han tendido a excluir situaciones intermedias. Sin embargo recientemente se están planteando las ventajas de superponer un suministro energético autosuficiente de pequeña escala con el suministro infraestructural. Este asunto hace que se comienza a debatir la conveniencia de un modelo híbrido de los dos anteriores ${ }^{\mathrm{ix}}$. Hasta ahora, cuando ha habido que planear el abastecimiento energético de un nuevo desarrollo urbano autosuficiente, la tendencia ha sido la de proponer sistemas de generación de energías renovables que pudieran devolver los excesos de energía generados a la red de suministro de mayor escala. Pero esta opción, que hasta el momento se ha considerado la idónea al ser rentable en base a subvenciones - por otro lado económicamente insostenibles en el tiempo- no es sin embargo la óptima desde el punto de vista de la eficiencia energética. Las fuentes de energía 
renovable de mediana y pequeña escala empleadas en estos desarrollos producen energía continua y, lamentablemente, en la transformación de corriente continua a alterna para su posterior introducción en el sistema se produce una pérdida de eficiencia de cerca del $25 \%^{\mathrm{x}}$ que hace que esta conversión sea un despilfarro energético. De modo similar, gran parte de los sistemas eléctricos empleados a escala doméstica - como pueden ser los ordenadores- funcionan con corriente continúa y al estar alimentados con corriente alterna, es necesario transformar el tipo de corriente, con la consecuente pérdida de un $10 \%$ xi de eficiencia en la transformación de alterna a continua. Por tanto dado que 1) la energía eléctrica de corriente alterna es más eficiente si se produce y distribuye de modo centralizado, y por el contrario el empleo de corriente continua es más eficientemente a menor escala, y dado que 2) ambos tipos de energía son necesarios en un desarrollo urbano, entonces podemos concluir que los dos modelos de urbanos antes descritos, el centralizado y el descentralizado -el conectado y el desconectado- no deben autoexcluirse sino convivir, debiendo emplearse de acuerdo con el fin al que estén destinados. En una vivienda cualquiera será más eficiente estar conectado tanto a la red de energía alterna centralizada para determinados usos como los frigoríficos $o$ las cocinas vitrocerámicas- como a la generación on-site de corriente continua mediante placas fotovoltaicas para el suministro de ordenadores personales o de paneles solares térmicos para el suministro de agua caliente sanitaria. Queda claro que el debate en torno a la energía y la ciudad no se puede limitar a la discusión acerca del grado de centralización o descentralización de las redes de suministro energético $\mathrm{o}$ al grado de conexión o desconexión con los sistemas de su entorno. La realidad es que existen pocas cuestiones inapelables, resultando necesario tener todas las opciones abiertas.

Sin embargo, sí es urgente que el debate en torno a ciudad y energía se haga partiendo de conceptos termodinámicos dado que, al fin y al cabo, están en la base del funcionamiento de las ciudades. Como es sabido las ciudades son sistemas termodinámicos abiertos $\mathrm{y}$, como tales, están modulados por las leyes de la termodinámica. Para conocer la realidad energética de una ciudad resulta necesario entender qué es y cómo funciona un sistema termodinámico abierto, cuál es la relación entre su estructura formal y su funcionamiento, y como afectan éstos intercambios de información a su consumo de materia y energía.

Las ciudades son sistemas abiertos conformados por una estructura formal concreta y unos ciudadanos, y el conjunto de relaciones -intercambios de información-que establecen entre todos estos. Este conjunto de relaciones no son independientes entre sí sino que forman una red de interacciones entre su estructura y su funcionamiento que, lejos de ser estables, están abiertos a variaciones en el tiempo. $\mathrm{Y}$ es esta variabilidad en el tiempo la hace que una ciudad tenga que reaccionar ante estos cambios, otorgándole la capacidad para reconfigurarse -auto-organizarsehacia estadios de mayor intercambio de información $\mathrm{y}$, por lo tanto, de mayor complejidad. El resultado es una organización interna más compleja que, como consecuencia, consume menos energía y materiales -son más eficientes- y genera menos entropía sobre los sistemas naturales de los que dependexii.

Es precisamente en este asunto en el que se centra el proyecto de investigación Syn-City desarrollado en el Imperial College de Londres y paradójicamente- financiado por British Petroleum (BP). El trabajo ha consistido en el desarrollo de tres modelos informáticos que tratan de conectar la relación existente entre una estructura urbana concreta - como por ejemplo un desarrollo urbano de baja densidad - y su consumo energético, analizando las conexiones entre una forma urbana y su comportamiento en el tiempo. El estúdio ha desarrollado un modelo que permite integrar sistemas de infraestructuras urbanas antes 
independientes - como por ejemplo las redes de transporte de metro o el caserío urbano- para encontrar sinergias termodinámicas que puedan existir entre ellas. La idea es encontrar situaciones urbanas en las que se den de modo simultáneo superávits y déficits de energía, de modo que las situaciones con exceso de energía puedan compensar las situaciones con carencia de energía, reduciendo la energía total que necesita la ciudad. El reto es interconectar en el espacio y el tiempo el funcionamiento termodinámico de las infraestructuras urbanas, de modo que cualquier situación que implique un superávit de energía pueda ser aprovechada por otra con déficit.

Este asunto ilumina el debate en torno a la ciudad y a la energía. Entendemos que lo importante no es ser pequeño y autosuficientes o tener 1oMW de energías renovables centralizadas especializarse- sino apostar por soluciones abiertas que potencien una progresiva acumulación de información que sea la base de una mejora en el empleo de la energía y la materia. Un

\section{Notas}

iDatos en World Energy Outlook 20o8, International Energy Agency: www.iea.org/textbase/nppdf/free/2008/weo 2008.pdf.

iiDatos en World Energy Outlook 20o8, International Energy Agency: www.iea.org/textbase/nppdf/free/2008/weo 2008.pdf.

iiiEstos y otros experimentos son descritos en el libro Sorry Out of Gas, editado por Mirko Zardini y Giovanna Borasi. 2008. Canadian Centre for Architecture, Montreal.

iv Ver el artículo de Andrés Jaque"Ecoaldeas frente a la Ciudad Eléctrica" publicado en El País el 6 de agosto de 2011 en funcionamiento interno más complejo es consecuencia de una acumulación de información, de conocimiento y de conexiones entre las cosas que potencia estructuras urbanas que, entre otras cosas, generan menos entropía.

La optimización es válida cuando existe un óptimo al que evolucionar. Por desgracia los sistemas abiertos están sometidos a flujos de materia, energía e información que provocan evoluciones en direcciones imprevistas. Por lo tanto no es tiempo de respuestas unívocas que nos conduzcan a callejones sin salida. Se debe apostar por modelos urbanos abiertos a la incorporación en tiempo real de nueva información que, desencadenando aumentos de la complejidad de funcionamiento del sistema, acaben por reducir la entropía que generan sobre los sistemas de los que depende.

http://elpais.com/diario/2011/o8/o6/babeli a/1312589570_850215.html.

vSe recomienda leer el libro La economía del Hidrógeno de Jeremy Rifkin. 2002. Paidós Estado y Sociedad, Barcelona (The Hydrogen Economy. 2002.Penguin Putnam Incorporation, New York.

viGeddes, Patrick. Cities in Evolution. 1915. Williams and Norgate Limited. London.

viiEs importante mencionar que la Unión Europea concedió al Gobierno de Navarra en 2003 el Premio a la Mejor Política Regional de Fomento de las Energías Renovables. 
viiihttp://www.navarra.es/home_es/Gobiern o+de+Navarra/Organigrama/Los+departam entos/Innovacion+Empresa+y+Empleo/Acci ones/Energia.htm

ixEsta tendencia ha quedado claramente enunciada en el artículo de Michelle Adlington "Energy Sub-structure, Suprastructure, Infra-structure" publicado en el libro Ecological Urbamism Mohsen Mostafavi con Gareth Doherty. 2010 Harvard University Graduate School of Design and Lars Müller Publishers.

xEstos datos son facilitados por Michelle Adlington en el artículo mencionado con anterioridad.

xiEstos datos son facilitados por Michelle Adlington en el artículo mencionado con anterioridad.

xiiPara ampliar estos conceptos consultar el libro de Ramón Margalef Perspectives in Ecological Theory. 1968. University of Chicago Press, Chicago. 